\title{
Clustering Based on the Node Health Status in Wireless Sensor Networks
}

\author{
A. Anhar ${ }^{1,2}$, R. Nilavalan ${ }^{1}$, Muhamad Syamsu Iqbal ${ }^{3}$ \\ ${ }^{1}$ Department of Electronic and Computer Engineering, Brunel University London, UB83PH, United Kingdom \\ Email: \{anhar.anhar, rajagopal.nilavalan\}@brunel.ac.uk \\ ${ }^{2}$ Department of Electrical Engineering, University of Riau, Pekanbaru, 28293, Indonesia \\ Email: anhar@lecturer.unri.ac.id \\ ${ }^{3}$ Department of Electrical Engineering, Universitas Mataram, Lombok, 83125, Indonesia \\ Email: msiqbal@unram.ac.id
}

\begin{abstract}
One of the applications of wireless sensor network is the forest fire monitoring which has different characteristics from others. In this application, the connectivity of nodes should not be destroyed just because of nodes lose their energy or burnt in the fire. Since the wide area of monitoring, the clustering method is considered as the efficient routing to increase its scalability as well as reduce energy consumed of nodes. Many clustering methods which are mostly based on Leach protocol are proposed without considering the node's failure. Here, we proposed the node health status as a parameter to select a Cluster Head and compared its performance with Leach, MTE and the direct algorithm. Results show that the number of packets received and the alive nodes of the m.nomosod method are higher than others. Identically, it has a........ end to end delay which is suitable for fores 1 application.
\end{abstract}

Leach;

Keywords-; clustering; health stat fort

\section{INTRI JCTION}

A forest fire becomes a $\mathrm{gl}$ fact th some effects which are raised by this activity. According to [1], the number of the burnt area increases from years to years, which is estimated between 300 and 400 million hectares per years. In addition, the spread of wildfire has influenced on the ecosystem, habitats, economics and biodiversity as a whole. The emission of carbon dioxide (CO2), haze and other greenhouse gases by fire give rise to the health issue and climate changes. For instance, in Indonesia, haze has been happening since 1982, and it becomes the unsolved problem which occurs every year [2]. Dry season which takes places in Indonesia precipitates wildfire beside other factors such as El Nino Southern Oscillation phenomenon, transmigration, and translation forest to commercial plantation e.g. of oil palms, using fire regarded as a cheaper and fastest than the mechanical technique for clearing land. Furthermore, Indonesia peatlands are huge carbon stores, and peatlands fire has a major contribution to the human health as well as the climate change in the world.

As stated in [3], there are three techniques to monitor forest fire: satellite-based system, an optical sensor and digital camera, and Wireless Sensor Network (WSN). WSN is a promising solution since it is easy to develop in the rural area, high robustness, and not influenced by weather or vision. It connects many nodes to sense some attributes of the environment and consists of two elements: node and gateway. Nodes in WSN are the main object of the network doing many functions such as sensing, processing/aggregating, and transmitting. Data or information that has been sensed by nodes is sent to Gateway or Base Station (BS) as a centre of processing and analysing data. Nodes in sensor network have varied in size and composed of four modules. A part that has a responsibility to sense parameters is called sensor module. Data or information then is sent to processing module before it is transmitted via th elesc communication module. In the clustering based $\mathrm{v}$ : less $\mathrm{s}$;or network, sensor nodes that become a Cluster lead ( I) perform aggregation in the wource suc s batt [4].

-so $1 \mathrm{~g}$ pro $: 0 \mathrm{lin} \mathrm{N}$ is application-specific. Due to very app tion 1 the di cent requirements, the design of uting col s ald cor ter these demands to a robust

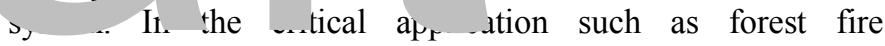
monitoring, when the fire spreads in the area, the fire can burn a sensor node which is close to the hotspot. As consequence, data from the sensor node will lose. In many clustering routing, a $\mathrm{CH}$ is chosen based on the residual energy, the Euclidian distance, or the random probability in order to enhance the energy consumed. However, they did not consider the death possibility of the selected $\mathrm{CH}$ because of fire. Choosing the safe $\mathrm{CH}$ will improve the robustness since there are no aggregation data loss in the network.

In this work, we propose the clustering technique according to the node health status. The node health status can be classified into safe and danger based on its distance to the hotspot. The proposed approach avoids to select the danger node in order to anticipate the loss of data from cluster member. The clustering routing which is grouping sensor nodes into clusters is applied in this work since it has been proposed by many researchers to enhance its scalability [5]. Also, clustering makes a simple routing table by localising route set up in every sensor nodes. Aggregating data in the $\mathrm{CH}$ to minimise relayed packets from sensor nodes to the Base station is another process which can reduce the energy consumed in a sensor network. 
This paper has been divided into four parts. The first part discusses the Leach and the routing for forest fire application. The proposed approach is presented in the second part. The third part discusses the parameters, scenarios and results of the simulation. Moreover, the final section is a conclusion and future work.

\section{RELATED WORKS}

Leach is cluster-based routing grouping sensor nodes in the cluster and is introduced by W. Heinzelman, et., all [6]. The operation of Leach is divided into rounds where every round consists of two phases: set-up phase and steady-state phase. In set-up phase, Leach randomly selects a few sensor nodes as $\mathrm{CHs}$ and other nodes which are near to the $\mathrm{CH}$ become its member. In the next round, the $\mathrm{CH}$ rotates among its member to evenly distribute the energy load among the sensors in the network. The steady-state operation is broken into frames, where nodes send their data to the $\mathrm{CH}$ at most once per frame during their allocated transmission slot. Each slot has a constant duration which depends on the number of nodes in the cluster. A TDMA schedule is used to minimize bandwidth, delay, and energy. Every $\mathrm{CH}$ has to stay awake in order to receive and aggregate the data from the sensor node. Leach assumes that all sensor nodes can directly transmit their data to the BS with enough power if needed and that the different Medium Access Control (MAC) techniques can be supported by every sensor node since they have the computational power. As consequent, Leach is not suitable to be applied to the network with large covera! armore, due to the concept of dynamic clusteri brings au nal overhead (head changes, advertisements limited energy of sensor nodes

TEEN and APTEEN are $\mathrm{t}$ proposed in [7][8]. These rout applications in the reactive ne TEEN, there are two threshol, al.), which y concumen by the $\mathrm{CH}$ to its member: Haru anu suil threshold. $v$ the other hand, APTEEN introduces both Hard/Soft Threshold and the query algorithm. The parameters of the environment are sensed by the sensor nodes continuously. If a certain parameter from the attribute set achieves its hard threshold value for the first time, the sensor node turns on its transmitter module and sends the packet to the BS. This packet is stored in the internal variable of the node as the sensed value (SV). The currently sensed attribute must be adjusted to the SV when the sensor node sends its packet. The weaknesses of TEEN is that the ability to send packet continuously even there is no packet which can achieve the threshold value while APTEEN has a complex algorithm.

A "context-aware" routing protocol that adapts its routing table based on the failure threat due to the sensed environments [9]. EMA proactively avoids route breakages due to node failure caused by the environment and at the same time attempts to be power efficient. EMA can provide robust and reliable data transfer from sources to the destination. A metric is used as the routing decision criteria to select the best neighbour to forward data. Nodes health status, RSSI, and hop count are parameters used to calculate the metric. Health status is defined to be a value between 0 and 100, with 0 being the worst and 100 the best health. Health status is linearly dependent on the temperature. If the temperature is below a detection threshold, the health conditions are 100. Otherwise, if the temperature is above the detection threshold, the health status is 0 , indicating that the node is likely to fail at any time. Even though EMA can provide a robust and reliable routing protocol, it is not suitable for monitoring fire since as the network grows, the end to end delay becomes bigger.

The author in [10] proposed a flat routing called Maximize Unsafe Path (MUP) which is optimizing the energy of node going to fail. The sensor node is classified into four grouped: safe, low safe, unsafe, and almost fail. Additionally, the Expected Transmission Count (ETX) defined as the approximate number of transmissions to send packets from a node to a destination successfully is a routing index to calculate the total cost of each route in the network. Again MUP introduces the high delay when the number of nodes increases.

Energy-efficient Fire Monitoring Protocol (EFMP) is a multi-hop hierarchical routing reorganizing clusters according to the environment [11]. The $\mathrm{CHs}$ are grouped into two categories: master and slave. A master $\mathrm{CH}$ is a $\mathrm{CH}$ which has the minimum number of transmission to the BS. If there are two or more $\mathrm{CHs}$ have the same amount of transmission, the amount of energy and the minimum number of node sensing the fire are other metrics specifying master $\mathrm{CH}$. There is only one master during a round. The slave $\mathrm{CHs}$ collect data from their members and send to the master $\mathrm{CH}$. Therefore, the amount of energy sumer; lower than existing clustering method such as $\mathrm{L}$ h. Ne theless, EFMP experiences the
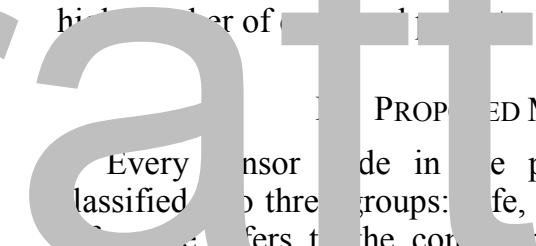
roups: fe, danger, and dead node. The while the dangerous node is the opposite one. Also, the node burnt off is known as a dead node. Fig. 1 shows the effect of selecting a dangerous node as a $\mathrm{CH}$. In (a), there is no fire around the cluster. On the contrary in (b), a selected $\mathrm{CH}$ is the dangerous node which is going to die at an unpredicted time. If the $\mathrm{CH}$ dies before it finishes transmitting data to the $\mathrm{BS}$, there will be many data loss.

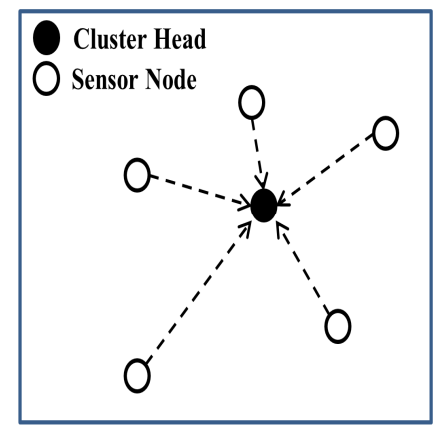

(a)

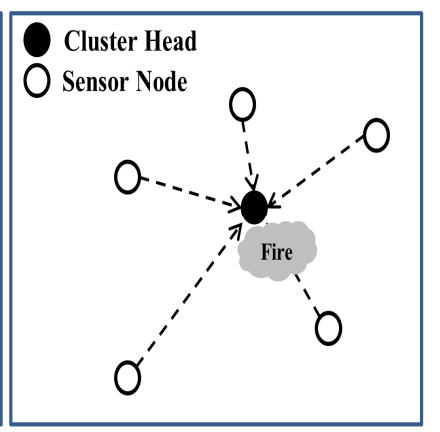

(b)
Fig. 1. Two different cases in choosing a $\mathrm{CH}$. 
Fig. 2 provides the steps in selecting $\mathrm{CH}$. It uses the same probability to become a $\mathrm{CH}$ as in Leach algorithm and adds another parameter, the node status. Every $\mathrm{CH}$ has to advertise its status to other sensor nodes. After receiving this information, sensor node selects the best $\mathrm{CH}$ based on its distance to the $\mathrm{CH}$. A dangerous node receiving advertisements from $\mathrm{CH}$ replies as soon as possible in order to get the priority in sending data. If there are two critical nodes in the different cluster, the priority will be given to the last sensor node detecting the fire.

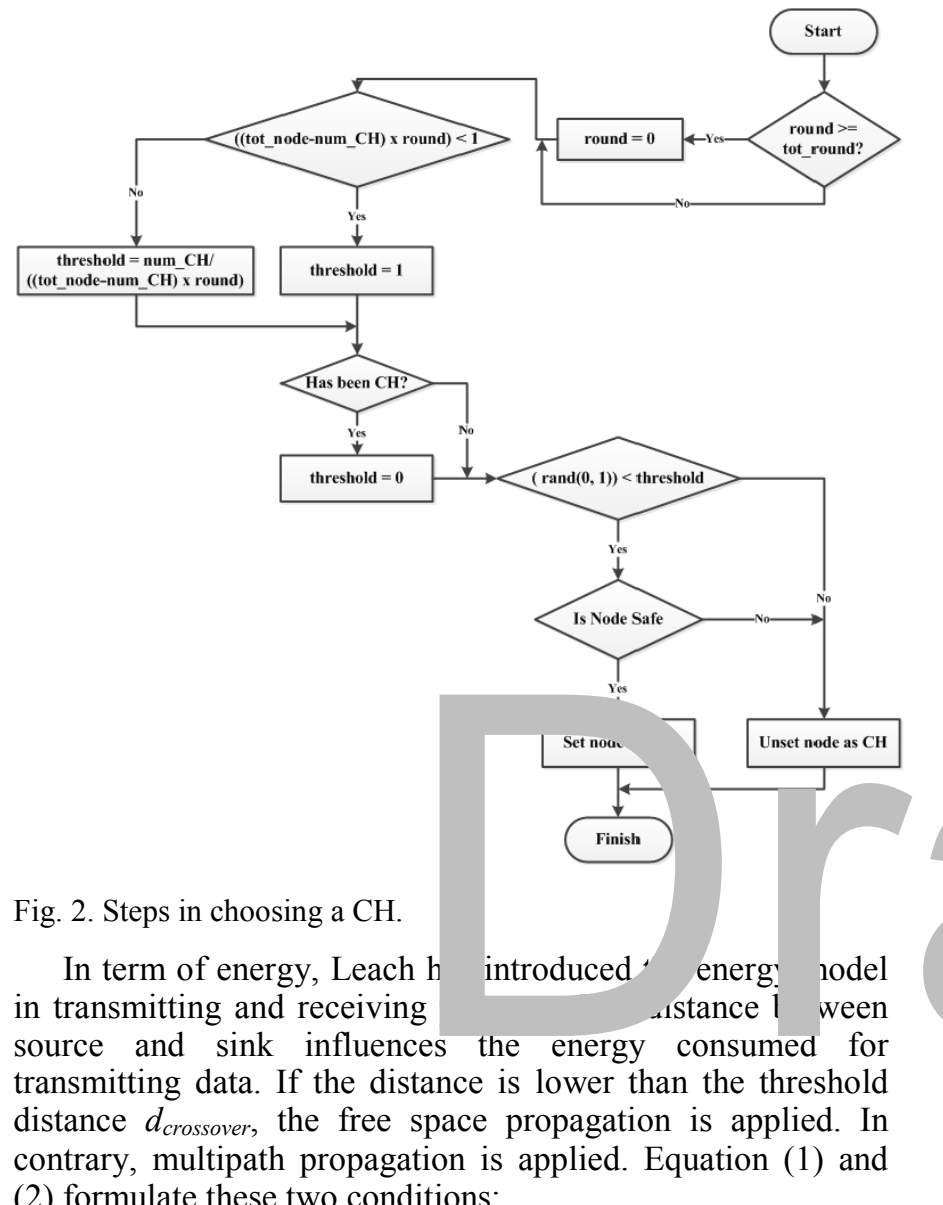

$$
\begin{gathered}
E_{T x}=l \cdot E_{\text {elect }}+l \cdot \varepsilon_{f s} \cdot d^{2} \text { for } 0 \leq d \leq d_{\text {crossover }} \\
E_{T x}=l \cdot E_{\text {elect }}+l \cdot \varepsilon_{m p} \cdot d^{4} \text { for } d>d_{\text {crossover }}
\end{gathered}
$$

Where $S_{\text {elect }}$ is energy consumed for electronic processing, $\varepsilon_{f s}$ and $\varepsilon_{M P}$ are constant for free space propagation and constant for multi-path propagation. On the other hand, energy spent in the receiver is:

$$
E_{R x}=l \cdot E_{\text {elect }}
$$

A communication channel in this simulation is symmetric therefore the same amount of energy is consumed to transmit data from sensor node to the $\mathrm{CH}$ or vice versa. To reduce the energy, a TDMA method is applied to send data from sensor member to the $\mathrm{CH}$. In addition, every cluster communicates using DSSS to reduce the interference between adjacent clusters. After receiving all data from its member, the $\mathrm{CH}$ senses the medium to check if other $\mathrm{CH}$ is sending data or not. If no other $\mathrm{CH}$ is sending data, the $\mathrm{CH}$ forwards data to $\mathrm{BS}$ using the BS spreading code.

\section{Simulation ScEnARIO AND RESUlts}

There are many platforms or tools or simulators can be used to study the performance of routing protocol in WSN. Most of them are Network Simulator (NS) 2, Malta platform, OPNET simulator, Noxim simulator, and IEEE 802.11 tool [12]. However, here we use Network Simulator (NS) 2.35 with the Leach protocol extension from MIT since we deal with not only the energy consumed but also the average end to end delay. The format of rca packet has to be modified and added the node health status in the header. NS is open source software, and it is a popular tool for the academic researchers. It supports many protocols in MAC and Network layer [13].

Table I present the simulation parameters in this simulation. Since we use Leach protocol as a benchmark, all parameters in table I have the same value as Leach routing. MAC sensor and TwoRayGround are the types of MAC layer and propagation. It assumes that every sensor node starts with the same level of energy and we place randomly a hundred nodes in the area $100 \times 100 \mathrm{~m} 2$. There is only one BS located at $50 ; 175$ with unlimited energy. The channel has the bandwidth 1 Mbps with delay $1 \times 10^{-12}$ second.

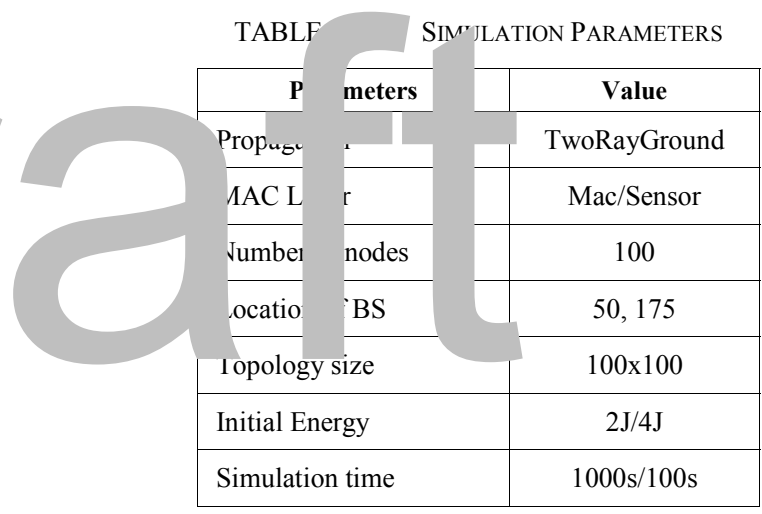

To measure the number of alive node and packets received, we run simulation 1000 second with initial energy 2J. At around the middle of the third round, one $\mathrm{CH}$ burned. Then another $\mathrm{CH}$ will be burned at around the middle of the ninth round. On the other hand, in term of end to end delay, we run only 100 second with initial energy $4 \mathrm{~J}$ in order to prevent some nodes run out of energy. We run the simulation with ten different topologies. The proposed approach is compared with Leach, MTE, and direct algorithm. MTE is a minimum transmission energy which is selecting the next-hop according to the closest node that is in the direction to the BS while the direct method is an algorithm to send data directly to the BS. 


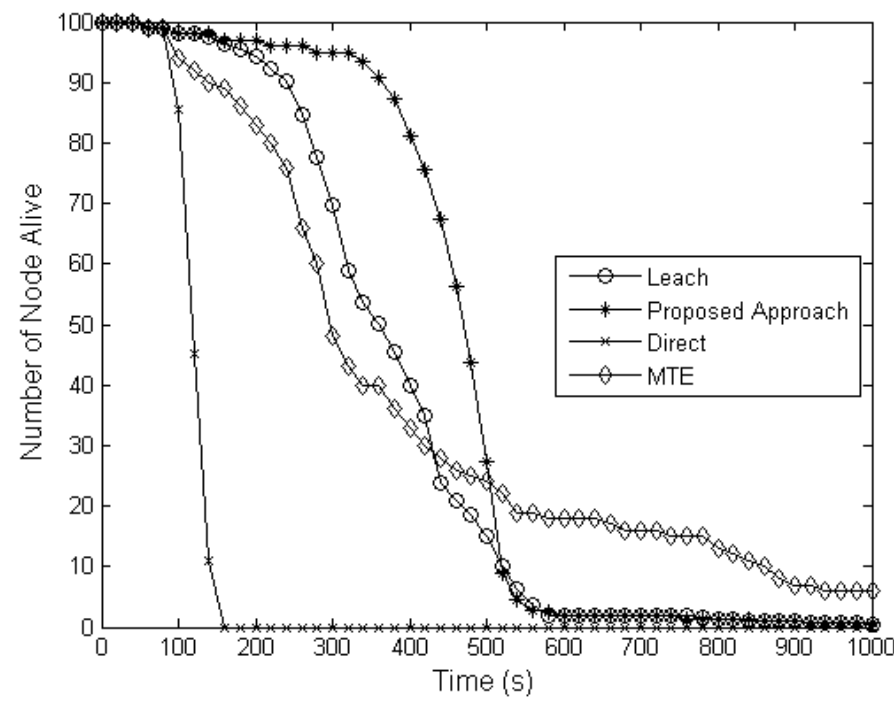

Fig. 3. The number of nodes alive v.s. time.

The number of nodes alive during simulation time is shown in Fig. 3. There is a sharp decrease in the number of dead node in direct method while the proposed approach, Leach, and MTE experience the same trend. At 100 second or the fifth round, only one node dies in the proposed approach and Leach whereas in MTE, there are five dead nodes. Furthermore, a half dead node in the proposed approach occurs at 460 seconds while in Leach and MTE, it happens at 360 and 300 seconds respectivelv. On the contrary, MTE has the longest network life time nsor node only transmits a little packet. Ther re still six 'es alive after 1000 second simulation time ir

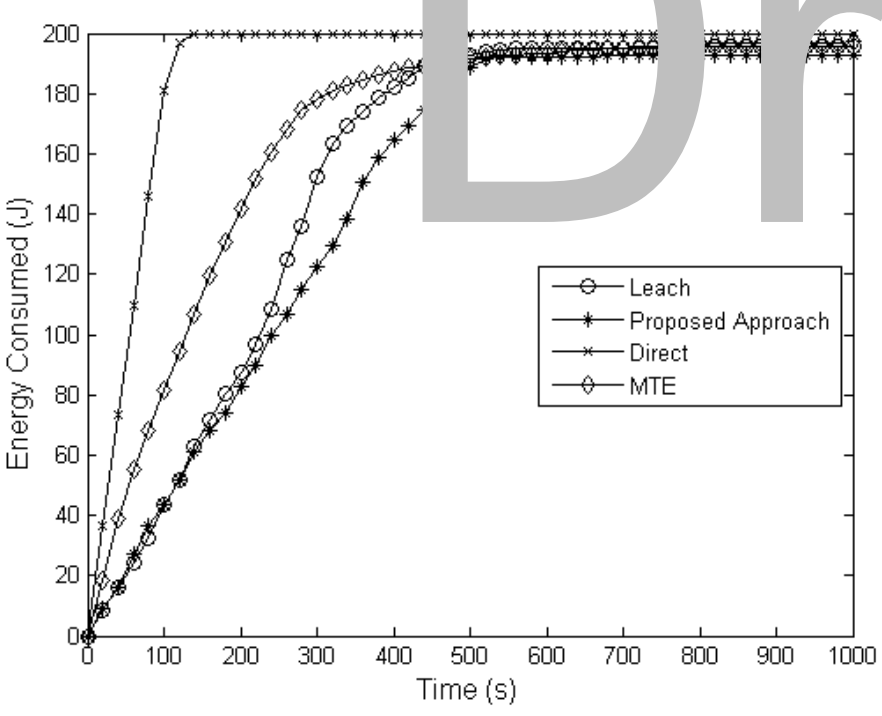

Fig. 4. Total energy consumed v.s. time.

Fig. 4 illustrates the amount of energy consumed over the simulation time. Overall, the proposed approach outperforms other algorithms. A half of total energy consumed in the proposed approach take place at around 260 seconds. On the other hand, Leach and MTE spend a hundred joule of energy at around 220 and 120 second successively. The worst case is in the direct method which is exhausting its energy at around 160 seconds.

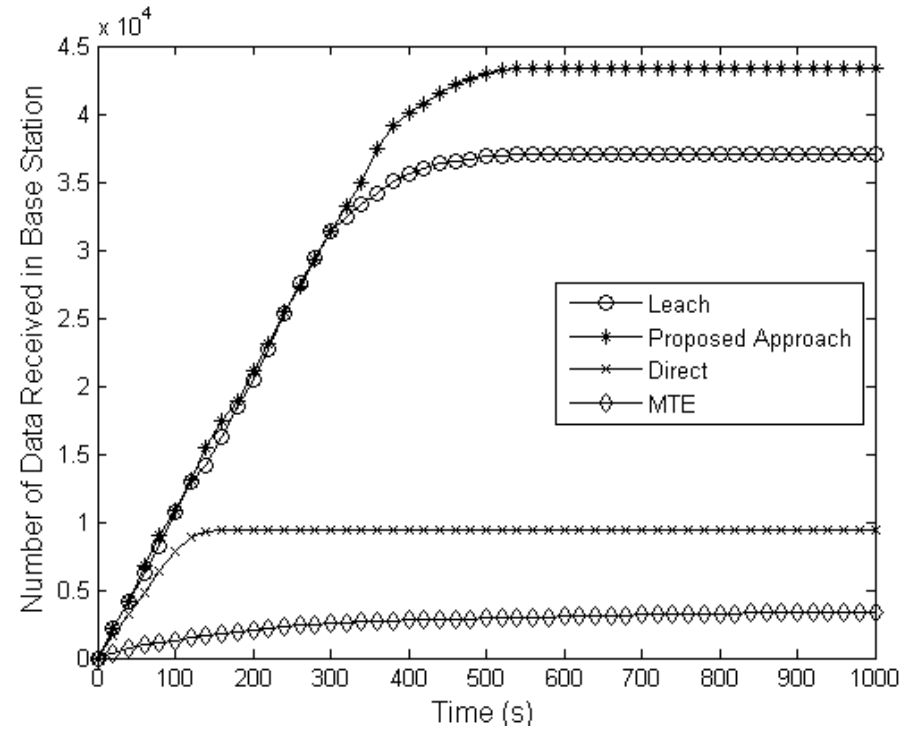

Fig. 5. The number of data received in BS v.s. time.

In term of data received, the proposed model has the superior performance as provided in Fig. 5. As the simulation time increases, the number of data received in the BS rises. The total data in the proposed approach after running 1000 second is approximately 43400 items while Leach, direct, and MTE achieve 37100, 9400, and 3365 packets serially. The highest number of data received in the proposed method is because this algorithm avoids choosing two dangerous nodes as $\mathrm{CH}$ although th bar lities fulfil the requirements of Leach method.

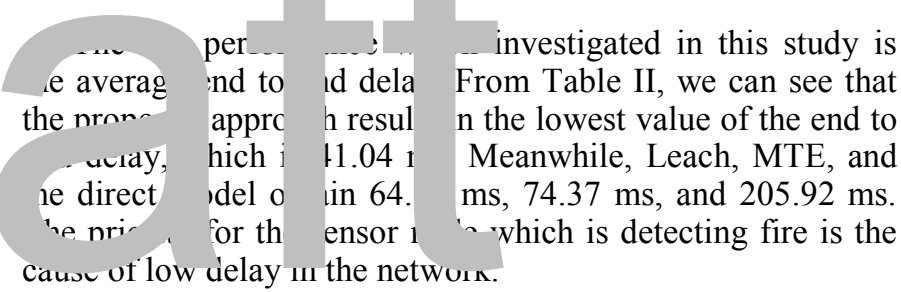

TABLE II. AVERAGE END To END DELAY

\begin{tabular}{|c|c|c|c|c|}
\hline & \multicolumn{4}{|c|}{ Protocol } \\
\cline { 2 - 5 } & Proposed Approach & Leach & MTE & Direct \\
\hline $\begin{array}{c}\text { Average Delay } \\
\text { (ms) }\end{array}$ & 41.05 & 64.72 & 94.37 & 205.92 \\
\hline
\end{tabular}

\section{CONCLUSION}

This paper aims to enhance the throughput as well as delay end to end of the wireless sensor network deployed for forest fire monitoring with considering the possibility of node failure because of burnt activities. We have developed the proposed approach which include the node health status as a variable to select the CHs.

In the future, dealing with the wide coverage of monitoring, the multi-hop routing scheme should be deployed to increase the network scalability. Furthermore, the impact of multi-hop communication to the average end-to-end delay of 
the network should be analysis since it may have played a vital role in the existence of fire.

\section{ACKNOWLEDGMENT}

The authors wish to thank the Directorate General of Resources for Science, Technology and Higher Education, the Ministry of Research, Technology and Higher Education of the Republic of Indonesia for providing A. Anhar a $\mathrm{PhD}$ scholarship to pursue his study at Brunel University London, UK.

\section{REFERENCES}

[1] E. Achyar, D. Schmidt-Vogt, and G. P. Shivakoti, "Dynamics of the multi-stakeholder forum and its effectiveness in promoting sustainable forest fire management practices in South Sumatra, Indonesia," Environ. Dev., vol. 13, pp. 4-17, 2015.

[2] W. J. de Groot, R. D. Field, M. A. Brady, O. Roswintiarti, and M. Mohamad, "Development of the Indonesian and Malaysian fire danger rating Systems," Mitig. Adapt. Strategy. Glob. Chang., vol. 12, no. 1, pp. 165-180, 2006.

[3] A. a. a. Alkhatib, "A review on forest fire detection techniques," Int. J. Distrib. Sens. Networks, vol. 2014, pp. 1-12, 2014.

[4] N. A. Pantazis, S. A. Nikolidakis, and D. D. Vergados, "Energy-efficient routino nrotocols in wireless sensor networks: A surve rmmun. Surv. TUTORIALS, vol. 15, 1 2, 2013.

[5] A. ABBASI and $\mathrm{N}$ YOUNIS, clustering algorithms Comput. Commun., 2841, 2007.

[6] W. B. Heinzelman, Balakrishnan, "An wireless sen net ks," 30, no. 14

P. Chand isan, d H. cific 1 tocol architecture for wireless microsensor networks," IEEE Trans. Wirel. Commun., vol. 1, no. 4, pp. 660-670,
2002.

[7] M. Ghiasabadi, M. Sharifi, N. Osati, S. Beheshti, and M. Sharifnejad, "TEEN: a routing protocol for enhanced efficiency in wireless sensor networks," 2008 Second Int. Conf. Futur. Gener. Commun. Netw., vol. 1, no. C, pp. 2009-2015, 2001.

[8] Manjeshwar A., D. P. Agrawal, and a Manjeshwar, "APTEEN: A hybrid protocol for efficient routing and comprehensive information retrieval in wireless sensor networks," Int. Parallel Distrib. Process. Symp., vol. 00, no. C, pp. 195-202, 2002.

[9] B.-L. Wenning, D. Pesch, A. Timm-Giel, and C. Görg, "Environmental monitoring aware routing: making environmental sensor networks more robust," Telecommun. Syst., vol. 43, no. 1-2, pp. 3-11, 2009.

[10] A. Jamil, D. J. Parish, R. C.-W. Phan, I. Phillips, J. Whitley, and G. Oikonomou, "Maximise unsafe path routing protocol for forest fire monitoring system using Wireless Sensor Networks," 2012 IEEE 3rd Int. Conf. Networked Embed. Syst. Every Appl., pp. 1-8, 2012.

[11] Y. G. Ha, H. Kim, and Y. C. Byun, "Energy-efficient fire monitoring over cluster-based wireless sensor networks," Int. J. Distrib. Sens. Networks, vol. 2012, 2012.

[12] A. Sarkar and T. Senthil Murugan, "Routing protocols for wireless sensor networks: What the literature says?," Alex ' a Eng. J., vol. 55, no. 4, pp. 31733183, 2016

[13] W. Kasch . War and J. Andrusenko, "Wireless an levelo is," IE Commun. Mag., vol. 47, no. 3, 20-12 2009.

+] T. ariya and E ossain, Introduction to Network $S$ lator JS) 2, `econd. Springer Science \& u ess N lia, 201. 